\title{
UNBOUNDED SOLUTION OF CHARACTERISTIC SINGULAR INTEGRAL EQUATION USING DIFFERENTIAL TRANSFORM METHOD
}

\author{
M. Abdulkawi ${ }^{1}$, M. Y. H. Akran ${ }^{2}$ \\ 1,2 Department of Mathematics, Faculty of Science and Arts, \\ Najran University, Saudi Arabia \\ 1Email: kawi2011@gmail.com
}

ABSTRACT: In this paper, The differential transform method is extended to solve the Cauchy type singular integral equation of the first kind. Unbounded solution of the Cauchy type singular Integral equation is discussed. Numerical results are shown to illustrate the efficiency and accuracy of the present solution.

2000 MSC: 65R20, 45E05

KEYWORDS: Singular integral equations; Cauchy kernel; Differential transform method.

\section{Council for Innovative Research}

Peer Review Research Publishing System

Journal: JOURNAL OF ADVANCES IN MATHEMATICS

Vol .9, No 7

www.ciriam.com, editoriam@gmail.com 


\section{INTRODUCTION:}

Consider the Cauchy type singular integral equation(CSIE) of the form

$$
\int_{-1}^{1} \frac{g(t)}{t-x} d t+\int_{-1}^{1} K(x, t) g(t) d t=f(x), \quad-1<\mathrm{x}<1,
$$

where $K(x, t)$ and $f(x)$ are given real valued functions belonging to the Holder class and $g(t)$ is to be determined, occurs in varieties of mixed boundary value problems of mathematical physics, isotropic elastic bodies involving cracks and other related problems [1-3]. The integral is considered as Cauchy principal value integral. Chakrabarti and Berge [4] have proposed an approximate method to solve CSIE (1) using polynomial approximation of degree $n$ and collocation points chosen to be the zeros of Chebyshev polynomial of the first kind for all cases. They showed that the approximate method is exact when the force function $f(t)$ is linear. Kim [5] solved CSIE by using Gaussian quadrature and chose the zeros of Chebyshev polynomials of the first and second kinds as the collocation and abscissa points. Abdulkawi [6] discussed the numerical solution of CSIE (1) for tow cases, unbounded and bounded,. He approximated the unknown function by weighted Chebyshev polynomials of the first and second kind, respectively, and used Lagrange-Chebyshev interpolation to approximate the regular kernel. Eshkuvatov et al.[7] discussed approximate solution of CSIE $(1)$ when $K(x, t)=0$ for four cases. They used weighted Chebyshev polynomials of the first, second, third and fourth kinds. They showed that the numerical solution is identical with the exact solution when the force function is a polynomial of degree one.

The characteristic CSIE is of the form

$$
\int_{-1}^{1} \frac{g(t)}{t-x} d t=f(x), \quad-1<\mathrm{x}<1 .
$$

It is known that the analytical solution of the equation (2) for unbounded case is given by the following expression [7]

$$
g(t)=-\frac{1}{\pi^{2} \sqrt{1-x^{2}}} \int_{-1}^{1} \frac{\sqrt{1-t^{2}} f(t)}{t-x} d t,
$$

where

$$
\int_{-1}^{1} g(t) d t=0
$$

\section{DIFFERENTIAL TRANSFORM METHOD}

The transformation of the $k$ th derivative of a function in one variable is as follows:

$$
F(k)=\frac{1}{k !}\left[\frac{d^{k} f(x)}{d x^{k}}\right]_{x=x_{0}}
$$

and the inverse transformation is defined by

$$
f(x)=\sum_{k=0}^{\infty} F(k)\left(x-x_{0}\right)^{k} .
$$

The following theorems can be deduced from Eqs. (5) and (6) [8].

Theorem 1. If $f(x)=g(x) \pm h(x)$, then

$$
F(k)=G(k) \pm H(k)
$$

Theorem 2. If $f(x)=a g(x)$, then $F(k)=a G(k)$, where $a$ is a constant and $G(k)$ is a differential transform of $g(x)$.

Theorem 3. If $f(x)=x^{n}$, then $F(k)=\delta(k-n)$, where 


$$
\delta(k-n)= \begin{cases}1, & k=n \\ 0, & k \neq n\end{cases}
$$

\section{THE SCHEME OF THE NUMERICAL SOLUTION}

The numerical solution of Eq. (2) is derived using the following procedures:

1- The unknown function $g(t)$ is written as:

$$
g(t)=\frac{\varphi(t)}{\sqrt{1-t^{2}}}
$$

where $\varphi(x)$ is regular function, so Eq. (2) becomes

$$
\int_{-1}^{1} \frac{\varphi(t)}{\sqrt{1-t^{2}}(t-x)} d t=f(x),-1<\mathrm{x}<1 .
$$

2- The following singular integrals are evaluated

$$
\text { 1) } \int_{-1}^{1} \frac{\varphi(t)}{\sqrt{1-t^{2}}(t-x)} d t, \quad-1<x<1 .
$$

$$
\text { 2) } \int_{-1}^{1} \frac{\varphi(t)}{\sqrt{1-t^{2}}} d t \text {. }
$$

3- The following condition is imposed to obtain the unique solution

$$
\int_{-1}^{1} \frac{\varphi(t)}{\sqrt{1-t^{2}}} d t=0
$$

\section{Theorem 4.}

If

$$
g(x)=\int_{-1}^{1} \frac{\varphi(t)}{\sqrt{1-t^{2}}(t-x)} d t
$$

Then the differential transform of $g(x)$ is

$$
G(m)=\sum_{k=1}^{N} \Phi(k) \sum_{r=0}^{k-1} C(k-r-1) \delta(m-r), \quad N \rightarrow \infty
$$

where $\Phi(k)$ is the differential transform of $\varphi(t)$, and

$$
C(k-r-1)= \begin{cases}\pi & \text { if } \quad k-r-1=0 \\ 0 & \text { if } \quad(k-r-1) \text { is odd } \\ \frac{\pi}{2^{k-r-1}}\left(\frac{k-r-1}{2}\right) & \text { if } \quad(k-r-1) \text { is even }\end{cases}
$$


Proof :

By using Maclaurin series

$$
\varphi(t)=\sum_{k=0}^{\infty} \Phi(k) t^{k}
$$

Rewriting $g(t)$ as follows

$$
\begin{aligned}
g(x) & =\int_{-1}^{1} \frac{\varphi(t)-\varphi(x)+\varphi(x)}{\sqrt{1-t^{2}}(t-x)} d t \\
& =\int_{-1}^{1} \frac{\varphi(t)-\varphi(x)}{\sqrt{1-t^{2}}(t-x)} d t+\int_{-1}^{1} \frac{\varphi(x)}{\sqrt{1-t^{2}}(t-x)} d t
\end{aligned}
$$

Using (12) into (13) yields

$$
\begin{aligned}
g(t)=\int_{-1}^{1} \frac{\sum_{k=0}^{\infty} \Phi(k) t^{k}-\sum_{k=0}^{\infty} \Phi(k) x^{k}}{\sqrt{1-t^{2}}(t-x)} d t+\int_{-1}^{1} \frac{\sum_{k=0}^{\infty} \Phi(k) x^{k}}{\sqrt{1-t^{2}}(t-x)} d t \\
=\sum_{k=1}^{\infty} \Phi(k) \int_{-1}^{1} \frac{t^{k}-x^{k}}{t-x} \cdot \frac{1}{\sqrt{1-t^{2}}} d t+\sum_{k=0}^{\infty} \Phi(k) x^{k} \int_{-1}^{1} \frac{d t}{\sqrt{1-t^{2}}(t-x)}
\end{aligned}
$$

It is known that

$$
\frac{t^{k}-x^{k}}{t-x}=\sum_{r=0}^{k-1} x^{r} \cdot t^{k-r-1}
$$

and [7]

$$
\int_{-1}^{1} \frac{d t}{\sqrt{1-t^{2}}(t-x)}=0
$$

Substituting (15) and (16) into (14) we get

$$
\begin{aligned}
g(x)=\sum_{k=1}^{\infty} \Phi(k) \int_{-1}^{1} \sum_{r=0}^{k-1} x^{r} \cdot \frac{t^{k-r-1}}{\sqrt{1-t^{2}}} d t \\
=\sum_{k=1}^{\infty} \Phi(k) \cdot \sum_{r=0}^{k-1} x^{r} \int_{-1}^{1} \frac{t^{k-r-1}}{\sqrt{1-t^{2}}} d t
\end{aligned}
$$

Let

$$
\int_{-1}^{1} \frac{t^{k-r-1}}{\sqrt{1-t^{2}}} d t=C(k-r-1)
$$

It is not difficult to see that 


$$
C(k)=\int_{-1}^{1} \frac{t^{k}}{\sqrt{1-t^{2}}} d t= \begin{cases}\pi & \text { if } \quad k=0, \\ 0 & \text { if } \quad k \text { is odd }, \\ \frac{\pi}{2^{k}}\left(\frac{k}{2}\right) & \text { if } \quad k \text { is even. }\end{cases}
$$

Due to (19) we get

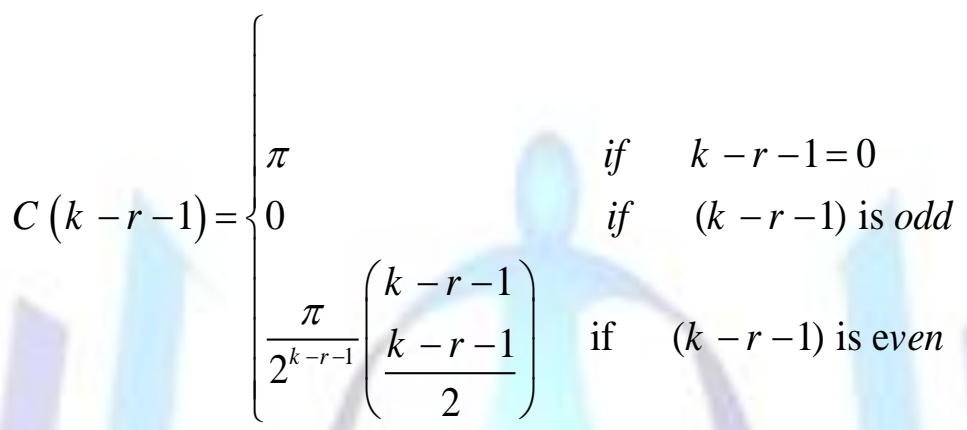

Hence

$$
g(x)=\sum_{k=1}^{N} \Phi(k) \cdot \sum_{r=0}^{k-1} x^{r} C(k-r-1)
$$

Taking the differential transform onto (21) and using theorems 1, 2 and 3 yields

$$
G(m)=\sum_{K=1}^{N} \Phi(k) \sum_{r=0}^{k-1} C(k-r-1) \delta(m-r), \quad N \rightarrow \infty
$$

The proof is completed.

\section{Theorem 5.}

If

$$
g(x)=\int_{-1}^{1} \frac{\varphi(t)}{\sqrt{1-t^{2}}} d t
$$

Then the differential transform of $g(x)$ is

$$
G(m)=\sum_{k=0}^{\infty} \Phi(k) C(k) \delta(m), \quad N \rightarrow \infty
$$

where $\Phi(k)$ is the differential transform of $\varphi(t)$, and C $(k)$ is defined by (19)

Proof :

Using (12) yields : 


$$
\begin{aligned}
g(x) & =\int_{-1}^{1} \frac{\sum_{k=0}^{\infty} \Phi(k) \cdot t^{k}}{\sqrt{1-t^{2}}} d t \\
& =\sum_{k=0}^{\infty} \Phi(k) \int_{-1}^{1} \frac{t^{k}}{\sqrt{1-t^{2}}} d t \\
& =\sum_{k=0}^{\infty} \Phi(k) \cdot C(k)
\end{aligned}
$$

Taking the differential transform onto (22) and using theorems 1,2 and 3 we obtain

$$
G(m)=\sum_{k=0}^{N} \Phi(k) \cdot C(k) \cdot \delta(m), \quad N \rightarrow \infty
$$

The proof is completed

\section{SOLUTION OF THE SYSTEM}

Taking the differential transform for two sides of Eq.(8) and using Theorem 4 yields

$$
\left.\begin{array}{c}
\sum_{K=1}^{N} \Phi(k) \sum_{r=0}^{k-1} C(k-r-1) \delta(m-r)=F(m), \\
m=0,1,2, \ldots ., N-1 .
\end{array}\right\}
$$

which equivalent to the following

$$
\begin{aligned}
m= & 0: \Phi(1) C(0)+\Phi(2) C(1)+\Phi(3) C(2)+\Phi(4) C(3)+\ldots+\Phi(N-3) C(N-4) \\
& +\Phi(N-2) C(N-3)+\Phi(N-1) C(N-2)+\Phi(N) C(N-1)=F(0) \\
m= & 1: \Phi(2) C(0)+\Phi(3) C(1)+\Phi(4) C(2)+\Phi(5) C(3)+\ldots+\Phi(N-3) C(N-5) \\
& +\Phi(N-2) C(N-4)+\Phi(N-1) C(N-3)+\Phi(N) C(N-2)=F(1) \\
m= & 2: \Phi(3) C(0)+\Phi(4) C(1)+\Phi(5) C(2)+\Phi(6) C(3)+\ldots+\Phi(N-3) C(N-6) \\
& +\Phi(N-2) C(N-5)+\Phi(N-1) C(N-4)+\Phi(N) C(N-3)=F(2) \\
m= & 3: \Phi(4) C(0)+\Phi(5) C(1)+\Phi(6) C(2)+\Phi(7) C(3)+\ldots+\Phi(N-3) C(N-7) \\
& +\Phi(N-2) C(N-6)+\Phi(N-1) C(N-5)+\Phi(N) C(N-4)=F(3) \\
m= & 4: \Phi(5) C(0)+\Phi(6) C(1)+\Phi(7) C(2)+\Phi(8) C(3)+\ldots+\Phi(N-3) C(N-8) \\
& +\Phi(N-2) C(N-7)+\Phi(N-1) C(N-6)+\Phi(N) C(N-5)=F(4) \\
& \vdots \\
m= & N-5: \Phi(N-4) C(0)+\Phi(N-3) C(1)+\Phi(N-2) C(2)+\Phi(N-1) C(3) \\
& +\Phi(N) C(4)=F(N-5) \\
m= & N-4: \Phi(N-3) C(0)+\Phi(N-2) C(1)+\Phi(N-1) C(2)+\Phi(N) C(3) \\
= & F(N-4) \\
m= & N-3: \Phi(N-2) C(0)+\Phi(N-1) C(1)+\Phi(N) C(2)=F(N-3) \\
m= & N-2: \Phi(N-1) C(0)+\Phi(N) C(1)=F(N-2) \\
m= & N-1: \Phi(N) C(0)=F(N-1)
\end{aligned}
$$

Applying Theorem 5 onto the condition (11), we have 


$$
G(m)=\sum_{k=0}^{N} \Phi(k) C(k) \delta(m)=0
$$

which gives the following equation

$\Phi(0) C(0)+\Phi(1) C(1)+\Phi(2) C(2)+. .+\Phi(N-1) C(N-1)+\Phi(N) C(N)=0$,

(25) From

(24) and (25) we obtain the following system of $(n+1) \times(n+1)$ for the unknown coefficients

$\Phi(k), k=0,1,2, \ldots, N$,

$$
\begin{aligned}
& \Phi(1) \pi+\Phi(3) C(2)+\Phi(5) C(4)+\Phi(7) C(6)+\ldots+\Phi(N-3) C(N-4) \\
& +\Phi(N-2) C(N-3)+\Phi(N-1) C(N-2)+\Phi(N) C(N-1)=F(0) \\
& \Phi(2) \pi+\Phi(4) C(2)+\Phi(6) C(4)+\Phi(8) C(6)+\ldots+\Phi(N-3) C(N-5) \\
& +\Phi(N-2) C(N-4)+\Phi(N-1) C(N-3)+\Phi(N) C(N-2)=F(1) \\
& \Phi(3) \pi+\Phi(5) C(2)+\Phi(7) C(4)+\Phi(9) C(6)+\ldots+\Phi(N-3) C(N-6) \\
& +\Phi(N-2) C(N-5)+\Phi(N-1) C(N-4)+\Phi(N) C(N-3)=F(2) \\
& \Phi(4) \pi+\Phi(6) C(2)+\Phi(8) C(4)+\Phi(10) C(6)+\ldots+\Phi(N-3) C(N-7) \\
& +\Phi(N-2) C(N-6)+\Phi(N-1) C(N-5)+\Phi(N) C(N-4)=F(3) \\
& \Phi(5) \pi+\Phi(7) C(2)+\Phi(9) C(4)+\Phi(11) C(6)+\ldots+\Phi(N-3) C(N-8) \\
& +\Phi(N-2) C(N-7)+\Phi(N-1) C(N-6)+\Phi(N) C(N-5)=F(4) \\
& \vdots \\
& \Phi(N-4) \pi+\Phi(N-2) C(2)+\Phi(N) C(4)=F(N-5) \\
& \Phi(N-3) \pi+\Phi(N-1) C(2)=F(N-4) \\
& \Phi(N-2) \pi+\Phi(N) C(2)=F(N-3) \\
& \Phi(N-1) \pi=F(N-2) \\
& \Phi(N) \pi=F(N-1) \\
& \Phi(0) \pi+\Phi(2) C(2)+\Phi(4) C(4)+\ldots .+\Phi(N-1) C(N-1)+\Phi(N) C(N)=0) \\
& \text { corresponding determinant is }
\end{aligned}
$$

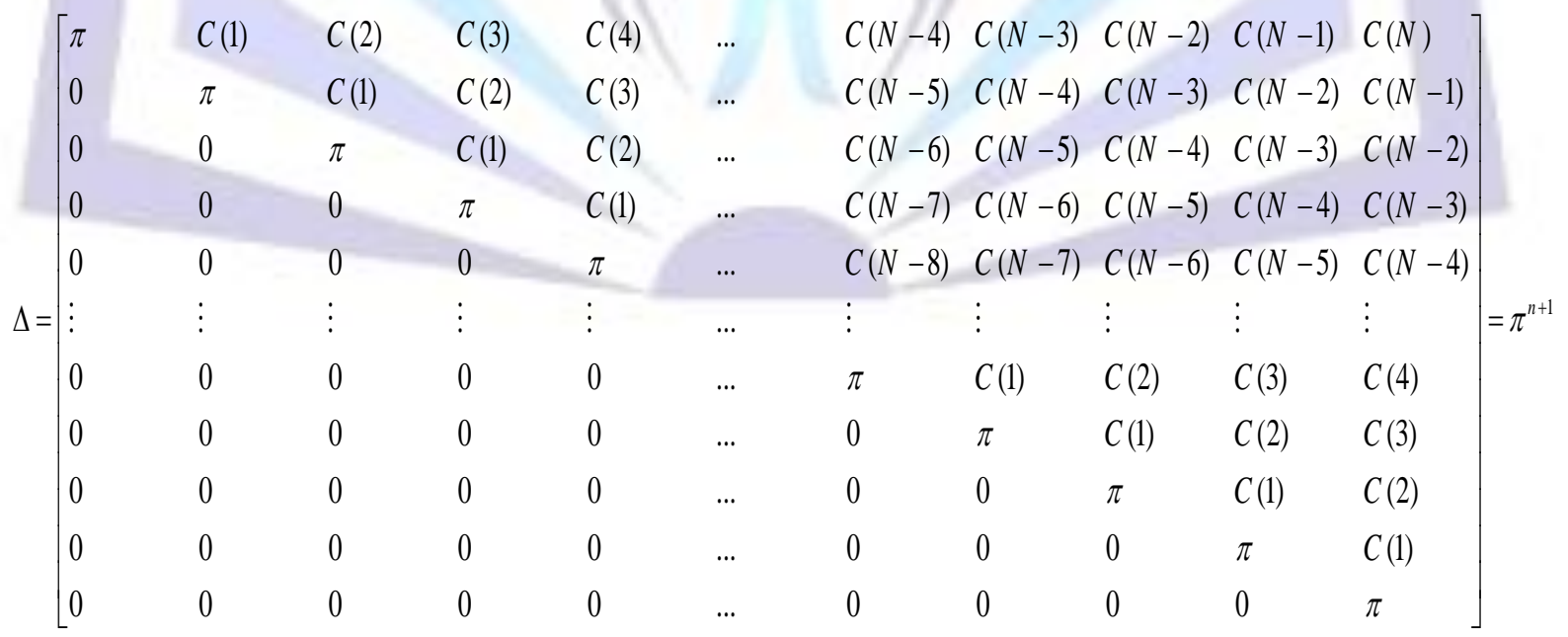

Since the determinant doesn't equal zero then the above system has a unique solution which is 


$$
\begin{aligned}
& \Phi(N)=\frac{1}{\pi} F(N-1) \\
& \Phi(N-1)=\frac{1}{\pi} F(N-2) \\
& \Phi(N-2)=\frac{1}{\pi}[F(N-3)-C(2) \Phi(N)] \\
& \Phi(N-3)=\frac{1}{\pi}[F(N-4)-C(2) \Phi(N-1)] \\
& \Phi(N-4)=\frac{1}{\pi}[F(N-5)-C(2) \Phi(N-2)-C(4) \Phi(N)] \\
& \Phi(N-5)=\frac{1}{\pi}[F(N-6)-C(2) \Phi(N-3)-C(4) \Phi(N-1)] \\
& \vdots \\
& \Phi(N-6)=\frac{1}{\pi}[F(N-7)-C(2) \Phi(N-4)-C(4) \Phi(N-2)-C(6) \Phi(N)] \\
& \Phi(N-7)=\frac{1}{\pi}[F(N-8)-C(2) \Phi(N-5)-C(4) \Phi(N-3)-C(6) \Phi(N-1)] \\
& \Phi(N-8)=\frac{1}{\pi}\left[\begin{array}{l}
F(N-9)-C(2) \Phi(N-6)-C(4) \Phi(N-4)-C(6) \Phi(N-2)] \\
-C(8) \Phi(N)
\end{array}\right] \\
& \Phi(N-9)=\frac{1}{\pi}\left[\begin{array}{l}
F(N-10)-C(2) \Phi(N-7)-C(4) \Phi(N-5)-C(6) \Phi(N-3)] \\
-C(8) \Phi(N-1)
\end{array}\right] \\
& =\frac{1}{\pi}\left[\begin{array}{l}
F(N-11)-C(2) \Phi(N-8)-C(4) \Phi(N-6)-C(6) \Phi(N-4)] \\
-C(8) \Phi(N-2)-C(10) \Phi(N)
\end{array}\right]
\end{aligned}
$$

Thus we obtain the general form of the numerical solution of equation (2) as follows

$$
\varphi(x)=\sum_{k=0}^{\infty} \Phi(k) x^{k}
$$

\section{NUMERICAL RESULTS}

The errors of the approximate solutions in the following examples are computed as the absolute value of the difference between the exact and approximate solutions.

Example 1. consider the following integral equation

$$
\int_{-1}^{1} \frac{\varphi(t)}{t-x} d t=x^{4}+5 x^{3}+2 x^{2}+x-\frac{11}{8} \quad, \quad-1<x<1
$$

Using the general formula of the exact solution given by (3) we obtain the exact solutions of Eq.(27) which is

$$
\varphi(x)=\frac{1}{\pi \sqrt{1-x^{2}}}\left[x^{5}+5 x^{4}+\frac{3}{2} x^{3}-\frac{3}{2} x^{2}-\frac{5}{2} x-\frac{9}{8}\right]
$$

It is clear that the differential transform of 


$$
f(x)=x^{4}+5 x^{3}+2 x^{2}+x-\frac{11}{8}
$$

is

$$
\left.\begin{array}{l}
F(0)=\frac{-11}{8} \\
F(1)=1 \\
F(2)=2 \\
F(3)=5 \\
F(4)=1 \\
F(k)=0, \quad k=5,6, \ldots
\end{array}\right\}
$$

From (26a) and (26b) for $N=5$ we obtain

$$
\begin{aligned}
& \Phi(5)=\frac{1}{\pi} F(4) \\
& \Phi(4)=\frac{1}{\pi} F(3) \\
& \Phi(3)=\frac{1}{\pi}[F(2)-C(2) \Phi(5)] \\
& \Phi(2)=\frac{1}{\pi}[F(1)-C(2) \Phi(4)] \\
& \Phi(1)=\frac{1}{\pi}[F(0)-C(2) \Phi(3)-C(4) \Phi(5)] \\
& \Phi(0)=\frac{1}{\pi}[-C(2) \Phi(2)-C(4) \Phi(4)]
\end{aligned}
$$

Using (19) and (29) into (30) we have

$$
\left.\begin{array}{l}
\Phi(0)=-\frac{9}{8 \pi}, \quad \Phi(1)=-\frac{5}{2 \pi}, \quad \Phi(2)=-\frac{3}{2 \pi}, \\
\Phi(3)=\frac{3}{2 \pi}, \quad \Phi(4)=\frac{5}{\pi}, \quad \Phi(5)=\frac{1}{\pi}
\end{array}\right\}
$$

Thus the numerical solution of Eq.(27) is

$$
\begin{aligned}
\varphi_{5}(x) & =\sum_{k=0}^{5} \varphi(k) x^{k} \\
& =\frac{1}{\pi}\left[-\frac{9}{8}-\frac{5}{2} x-\frac{3}{2} x^{2}+\frac{3}{2} x^{3}+5 x^{4}+x^{5}\right]
\end{aligned}
$$

which is identical to the exact solution. 


\begin{tabular}{|c|c|c|}
\hline \multirow{2}{*}{ Table 1. Comparison of errors between method in [7] and proposed method for Eq.(27). } \\
\cline { 2 - 3 } & \multicolumn{2}{|c|}{ Errors } \\
\cline { 2 - 3 } & Method in [7] $(N=20)$ & Proposed method $(N=5)$ \\
\hline-0.95 & $8.881784197001252 \mathrm{E}-016$ & $0.00000000000000 \mathrm{E}-000$ \\
\hline-0.90 & $-6.661338147750939 \mathrm{E}-016$ & $0.00000000000000 \mathrm{E}-000$ \\
\hline-0.70 & $7.494005416219807 \mathrm{E}-016$ & $0.00000000000000 \mathrm{E}-000$ \\
\hline-0.50 & $-5.551115123125783 \mathrm{E}-016$ & $0.00000000000000 \mathrm{E}-000$ \\
\hline-0.30 & $4.163336342344337 \mathrm{E}-016$ & $0.00000000000000 \mathrm{E}-000$ \\
\hline-0.10 & $-3.330669073875470 \mathrm{E}-016$ & $0.00000000000000 \mathrm{E}-000$ \\
\hline 0.00 & $1.665334536937735 \mathrm{E}-016$ & $0.00000000000000 \mathrm{E}-000$ \\
\hline 0.10 & $5.551115123125783 \mathrm{E}-016$ & $0.00000000000000 \mathrm{E}-000$ \\
\hline 0.30 & $0.000000000000000 \mathrm{E}-000$ & $0.00000000000000 \mathrm{E}-000$ \\
\hline 0.50 & $-7.771561172376096 \mathrm{E}-016$ & $0.00000000000000 \mathrm{E}-000$ \\
\hline 0.70 & $0.000000000000000 \mathrm{E}-000$ & $0.00000000000000 \mathrm{E}-000$ \\
\hline 0.90 & $9.436895709313831 \mathrm{E}-016$ & $0.000000000000000 \mathrm{E}-00$ \\
\hline 0.95 & $8.881784197001252 \mathrm{E}-016$ & $0.00000000000000 \mathrm{E}-000$ \\
\hline & & \\
\hline
\end{tabular}

Example 2. consider the following singular integral equation

$$
\int_{-1}^{1} \frac{\varphi(t)}{\sqrt{1-t^{2}}(t-x)} d t=4 x^{2}-1
$$

It is clear that the differential Transform of

$$
f(x)=4 x^{2}-1
$$

is

$$
\left.\begin{array}{l}
F(0)=-1, \quad F(1)=0 \\
F(2)=4, \quad F(k)=0, \quad k=3,4, \ldots
\end{array}\right\}
$$

From (26a) and (26b) for $N=3$ we obtain

$$
\left.\begin{array}{l}
\Phi(3)=\frac{1}{\pi} F(2) \\
\Phi(2)=\frac{1}{\pi} F(1) \\
\Phi(1)=\frac{1}{\pi}[F(0)-C(2) \Phi(3)] \\
\Phi(0)=\frac{1}{\pi}[-C(2) \Phi(2)]
\end{array}\right\}
$$

Using (19) and (32) into (33) we have 


$$
\left.\begin{array}{l}
\Phi(0)=0, \quad \Phi(1)=-\frac{3}{\pi}, \\
\Phi(2)=0, \quad \Phi(3)=\frac{4}{\pi} .
\end{array}\right\}
$$

Thus the numerical solution of Eq.(31) is

$$
\begin{aligned}
\varphi_{3}(x) & =\sum_{k=0}^{3} \varphi(k) x^{k} \\
& =\left[-\frac{3}{\pi} x+\frac{4}{\pi} x^{3}\right] \\
& =\frac{1}{\pi}\left[-3 x+4 x^{3}\right]
\end{aligned}
$$

\begin{tabular}{|c|c|c|c|}
\hline \multirow[t]{2}{*}{$x$} & \multirow{2}{*}{ Exact } & \multicolumn{2}{|c|}{ Numerical solution } \\
\hline & & $\begin{array}{l}\text { Method in [9] } \\
\qquad(N=65)\end{array}$ & $\begin{array}{c}\text { Proposed } \\
\text { method } \\
(N=3)\end{array}$ \\
\hline 0 & 0 & $-1.22 \mathrm{E}-14$ & 0 \\
\hline 0.25 & -0.68750 & -0.68798 & -0.68750 \\
\hline 0.50 & -1.00000 & -1.00096 & -1.00000 \\
\hline 0.75 & -0.56250 & -0.56395 & -0.56250 \\
\hline
\end{tabular}

which is identical to the exact solution.

Example 3. Consider the following singular integral equation

$$
\int_{-1}^{1} \frac{\varphi(t)}{\sqrt{1-t^{2}}(t-x)}=x^{10}
$$

It is clear that the differential Transform of

$$
f(x)=x^{10}
$$

is

$$
\left.\begin{array}{l}
F(10)=1 \\
F(k)=0, \quad k \neq 10 .
\end{array}\right\}
$$

From (26a) and (26b) for $N=11$ and using (36) we obtain

$$
\begin{aligned}
\Phi(11) & =\frac{1}{\pi} F(10)=\frac{1}{\pi} \\
\Phi(10) & =\frac{1}{\pi} F(9)=0 \\
\Phi(9) & =\frac{1}{\pi}[F(8)-C(2) \Phi(11)]=-\frac{1}{2 \pi}
\end{aligned}
$$




$$
\begin{aligned}
\Phi(8) & =\frac{1}{\pi}[F(7)-C(2) \Phi(10)]=0 \\
\Phi(7) & =\frac{1}{\pi}[F(6)-C(2) \Phi(9)-C(4) \Phi(11)] \\
& =\frac{1}{4 \pi}-\frac{3}{8 \pi}=\frac{-1}{8 \pi} \\
\Phi(6) & =\frac{1}{\pi}[F(5)-C(2) \Phi(8)-C(4) \Phi(10)]=0 \\
\Phi(5) & =\frac{1}{\pi}[F(4)-C(2) \Phi(7)-C(4) \Phi(9)-C(6) \Phi(11)] \\
& =-\frac{1}{8 \pi}+\frac{3}{8 \pi}-\frac{5}{16 \pi}=-\frac{1}{16 \pi} \\
\Phi(4) & =\frac{1}{\pi}[F(3)-C(2) \Phi(6)-C(4) \Phi(8)-C(6) \Phi(10)]=0 \\
\Phi(3) & =\frac{1}{\pi}[F(2)-C(2) \Phi(5)-C(4) \Phi(7)-C(6) \Phi(9)-C(8) \Phi(11)] \\
& =\frac{1}{16 \pi}-\frac{9}{32 \pi}+\frac{5}{16 \pi}+\frac{9}{64 \pi}-\frac{35}{128 \pi}=\frac{-5}{128 \pi} \\
\Phi(2) & =\frac{1}{\pi}[F(1)-C(2) \Phi(4)-C(4) \Phi(6)-C(6) \Phi(8)-C(8) \Phi(10)]=0 \\
& =-\frac{1}{32 \pi}+\frac{3}{16 \pi}-\frac{15}{64 \pi}-\frac{27}{128 \pi}+\frac{15}{64 \pi}+\frac{35}{128 \pi}-\frac{63}{256 \pi}=\frac{-7}{256 \pi} \\
&
\end{aligned}
$$

Thus the numerical solution of Eq.(35) is

$$
\varphi_{11}(x)=\sum_{k=0}^{11} \Phi(k) x^{k}=\frac{1}{\pi}\left[-\frac{7}{256} x-\frac{5}{128} x^{3}-\frac{1}{16} x^{5}-\frac{1}{8} x^{7}-\frac{1}{2} x^{9}+x^{11}\right]
$$

which is identical to the exact solution.

\section{CONCLUSION}

We have developed an efficient approximate method for solving the characteristic singular integral equation with Cauchy kernel for the unbounded case. The developed approximate method gives a very accurate numerical results with only small number of knot points $N$. These show that our approximate method gives a very accurate numerical solution to the characteristic Cauchy type singular integral equation. Moreover, our approximate method gives the exact solution when the force function $f(x)$ is a polynomial of higher degree.

\section{REFERENCES}

[1] Gakhov, F.D., 1963. Boundary value problems. Translation edited by Sneddon, I.N. Pergamon Press Ltd. 
[2] Lifanov, I.K., 1996. Singular Integral Equation and Discrete Vortices. VSO, The Netherlands.

[3] Ladopoulos, E.G., 2000. Singular integral equations, Linear and Non-Linear, Theory and its applications in Science and Engineering. Springer-Verlag.

[4] Chakrabarti, A. and Berghe, V.G., Approximate Solution of Singular Integral Equations. Appl. Math. Lett. 17(2004), 553-559.

[5] Kim, S. Solving singular integral equations using Gaussian quadrature and over determined system. Appl. Math. Comput, 35 (1998), 63-71.

[6] Abdulkawi, M. A Numerical Solution of Singular Integral Equations. International Journal of Mathematics and Statistics, Volume 10, Number W11(2011), 69-76.

[7] Eshkuvatov, Z.K., Nik Long, N. M. A. and Abdulkawi, M. Approximate solution of singular integral equations of the first kind with Cauchy kernel. Appl. Math. Lett, 22 (2009), 651-657.

[8] Aytac Arikoglu and Ibrahim Ozkol. Solutions of integral and integro-differential equation systems by using differential transform method. Computers and Mathematics with Applications, 56(2008) 2411-2417.

[9] Gerasoulis, A. and Srivastava, R. P. A Method for the numerical solution of singular integral equations with a principal value integral. Int. J. Engng sci. Vol. 19, (1981), 1293-1298. 